\title{
A Robust Estimation to Transmission Line Parameters Based on Huber Estimation with PMU data
}

\author{
Yanjun Zhao ${ }^{1,}$,, Xiaoke Chen ${ }^{1, \text { a }}$, Yue Zhang ${ }^{1, \text { a }}$, \\ Zihua Huang ${ }^{2, b}$, Ancheng Xue 2 , b \\ ${ }^{1}$ Electric Power Research Institute of Guangdong Power Grid Co. LTD, Guangzhou, Guangdong \\ Province 510080, China \\ ${ }^{2}$ North China Electric Power University State Key Laboratory of Alternate Electrical Power System \\ With Renewable Energy Sources, Changping District, Beijing 102206, China \\ aemail: zhaoyanjun_ceeer@126.com, bemail:shinichi@ncepu.edu.cn
}

Keywords: Transmission line; parameter identification; robust least square; Huber Estimation; PMU data

\begin{abstract}
In the parameter identification for transmission line, there often exist the gross errors in the measured data and lacking of robustness with traditional least square. This paper applies the Huber estimation to the transmission line parameter estimation, i.e., proposes a robust least square estimation to line parameter identification based on Huber estimation. The simulation results based on PSCAD demonstrate the effectiveness and robust ability of the proposed method.
\end{abstract}

\section{Introduction}

The accurate transmission line parameters are the basis of power flow calculation, state estimation (SE) and other advanced applications of EMS in electric power system engineering. Currently, in power system analysis, the transmission line parameters often use the design parameters or the experimental parameters obtained at certain condition, rather than the actual or on-line parameters. It may affect the other security analysis. Thus, it is important to develop an effective transmission line parameter estimation method ${ }^{[1]}$.

In general, the on-line transmission line parameter estimation methods could be classified to two categories, one is the methods irrelevant to $\mathrm{SE}^{[2,3]}$ and the other is the methods related to $\mathrm{SE}^{[4-9] \text {. }}$

Currently, with the development of phasor measurement unit (PMU) and wide area measurement system (WAMS) technology ${ }^{[10]}$, which provides a powerful data source to on-line parameter identification of transmission line, a lot of on-line identification methods have been proposed ${ }^{[4-9]}$.

However, as the PMU data may have error, the proposed method may give out not good result. Recognizing the above, this paper proposes a robust on-line identification method for transmission line parameter based on the Huber estimation.

The proposed method formulates the identification of line parameter as an optimization problem. However, in the objective function, the Huber estimation, which is a robust estimation, is adopted. The simulation results based on PSCAD demonstrate the effectiveness, noise immunity ability and robust ability of the proposed method.

The remainders of this paper are organized as follows. In section II, the mathematical model for the transmission line is presented. In section III and section IV, the traditional LS and the RLS based on Huber estimation are introduced. And in section V, the simulation results with PSCAD are presented. Finally, the conclusions are remarked in section VI.

\section{Augmented based on WLS}

This section presents the mathematical formulation transmission line. The transmission line adopted the $\pi$-type equivalent circuit as show in Fig.1. 


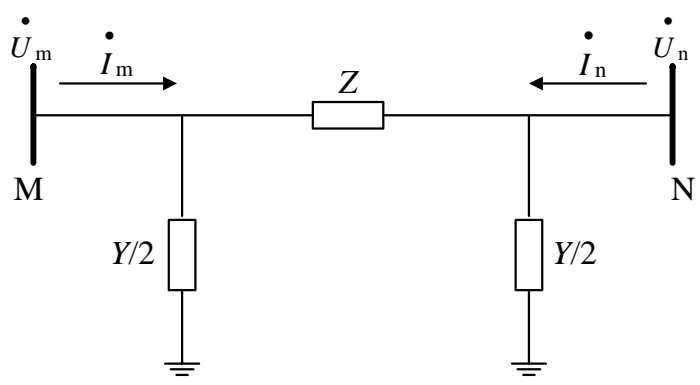

Fig.1. $\pi$-type equivalent circuit

With the PMU data obtained at the two terminals of the line, the following equation can be obtained.

$$
\left[\begin{array}{l}
\dot{I}_{\mathrm{m}} \\
\dot{I}_{\mathrm{n}} \\
\dot{S}_{\mathrm{m}} \\
\dot{S}_{\mathrm{n}}
\end{array}\right]=\left[\begin{array}{cc}
\dot{U}_{\mathrm{m}}-\dot{U}_{\mathrm{n}} & \dot{U}_{\mathrm{m}} \\
\dot{U}_{\mathrm{n}}-\dot{U}_{\mathrm{m}} & \dot{U}_{\mathrm{n}} \\
U_{\mathrm{m}}^{2}-\dot{U}_{\mathrm{m}} \dot{U}_{\mathrm{n}} & U_{\mathrm{m}}^{2} \\
U_{\mathrm{n}}^{2}-\stackrel{*}{U}_{\mathrm{n}} \dot{U}_{\mathrm{m}} & U_{\mathrm{n}}^{2}
\end{array}\right]\left[\begin{array}{c}
1 / Z \\
Y / 2
\end{array}\right]
$$

where $Z=R+\mathrm{j} X, Y=\mathrm{j} B$ is the reactance and susceptance of the transmission line, $B=2 \pi f C, C$ is the capacity $f=50 \mathrm{~Hz}$. With $\operatorname{Eq}(1)$, the following can be obtained.

$$
\left[\begin{array}{l}
I_{\mathrm{mR}} \\
I_{\mathrm{mI}} \\
I_{\mathrm{nR}} \\
I_{\mathrm{nI}} \\
P_{\mathrm{m}} \\
Q_{\mathrm{m}} \\
P_{\mathrm{n}} \\
Q_{\mathrm{n}}
\end{array}\right]=\left[\begin{array}{lcc}
U_{\mathrm{m}} \cos \theta_{\mathrm{um}}-U_{\mathrm{n}} \cos \theta_{\mathrm{un}} & U_{\mathrm{n}} \sin \theta_{\mathrm{un}}-U_{\mathrm{m}} \sin \theta_{\mathrm{um}} & -U_{\mathrm{m}} \sin \theta_{\mathrm{um}} \\
U_{\mathrm{m}} \sin \theta_{\mathrm{um}}-U_{\mathrm{n}} \sin \theta_{\mathrm{un}} & U_{\mathrm{m}} \cos \theta_{\mathrm{um}}-U_{\mathrm{n}} \cos \theta_{\mathrm{un}} & U_{\mathrm{m}} \cos \theta_{\mathrm{um}} \\
U_{\mathrm{n}} \cos \theta_{\mathrm{un}}-U_{\mathrm{m}} \cos \theta_{\mathrm{um}} & U_{\mathrm{m}} \sin \theta_{\mathrm{um}}-U_{\mathrm{n}} \sin \theta_{\mathrm{un}} & -U_{\mathrm{n}} \sin \theta_{\mathrm{un}} \\
U_{\mathrm{n}} \sin \theta_{\mathrm{un}}-U_{\mathrm{m}} \sin \theta_{\mathrm{um}} & U_{\mathrm{n}} \cos \theta_{\mathrm{un}}-U_{\mathrm{m}} \cos \theta_{\mathrm{um}} & U_{\mathrm{n}} \cos \theta_{\mathrm{un}} \\
U_{\mathrm{m}}{ }^{2}-U_{\mathrm{m}} U_{\mathrm{n}} \cos \left(\theta_{\mathrm{um}}-\theta_{\mathrm{un}}\right) & -U_{\mathrm{m}} U_{\mathrm{n}} \sin \left(\theta_{\mathrm{um}}-\theta_{\mathrm{un}}\right) & 0 \\
-U_{\mathrm{m}} U_{\mathrm{n}} \sin \left(\theta_{\mathrm{um}}-\theta_{\mathrm{un}}\right) & U_{\mathrm{m}} U_{\mathrm{n}} \cos \left(\theta_{\mathrm{um}}-\theta_{\mathrm{un}}\right)-U_{\mathrm{m}}{ }^{2} & -U_{\mathrm{m}}{ }^{2} \\
U_{\mathrm{n}}{ }^{2}-U_{\mathrm{n}} U_{\mathrm{m}} \cos \left(\theta_{\mathrm{un}}-\theta_{\mathrm{um}}\right) & -U_{\mathrm{n}} U_{\mathrm{m}} \sin \left(\theta_{\mathrm{un}}-\theta_{\mathrm{um}}\right) & 0 \\
-U_{\mathrm{n}} U_{\mathrm{m}} \sin \left(\theta_{\mathrm{un}}-\theta_{\mathrm{um}}\right) & U_{\mathrm{n}} U_{\mathrm{m}} \cos \left(\theta_{\mathrm{un}}-\theta_{\mathrm{um}}\right)-U_{\mathrm{n}}{ }^{2} & -U_{\mathrm{n}}{ }^{2}
\end{array}\right]\left[\begin{array}{l}
g \\
b \\
y_{\mathrm{c}}
\end{array}\right]
$$

where the $I_{\mathrm{mR}}, I_{\mathrm{mI}}, I_{\mathrm{nR}}, I_{\mathrm{nI}}$ are the real and imaginary part of the current, $U_{\mathrm{m}}, \theta_{\mathrm{um}}, U_{\mathrm{n}}$, $\theta_{\mathrm{un}}$ are the voltage magnitude and phase, $P_{\mathrm{m}}, Q_{\mathrm{m}}, P_{\mathrm{n}}, Q_{\mathrm{n}} g, b$ is the real and imagariy part of $1 / Z, y_{c}$ is the imaginary of $Y / 2$.

In considering the error of the PMU, the $\mathrm{Eq}(2)$ can be written as follows.

$\beta=A \alpha+v$

where $\boldsymbol{\alpha}=\left[\begin{array}{lll}g & b & y_{\mathrm{c}}\end{array}\right]^{\mathrm{T}}$ are the parameters to be estimated and the $\boldsymbol{v}$ is the residue error vector.

\section{Traditional least square estimation}

The traditional least square (TLS) estimation uses the sum of the square of the difference as the objective function. In general, the parameter identification for $\boldsymbol{\alpha}$ could be described as the following optimization problem.

$$
\min J(\alpha)=\sum_{i=1}^{N} P_{i} v_{i}^{2}
$$

where the $\mathrm{N}$ is the number of the samples. $P_{i}$ and $v_{i}$ are the weigh and residue of the No.i data.

The TLS is based on the assumption that the measured data obeys the normal distribution. So it uses the same weights. Thus it may give out wrong result as the, as the measure data may exist grosss error, which could strongly impact the objective function. In recognizing the above, this paper presents the Huber estimation to reduce the impact of the gross data and bad data. 


\section{Robust Least Squares Estimation}

This section presents the essence of the robust Huber estimation and then applies it to the identification.

A. Huber Estimation

Large number of practice has proved that the measured data which strictly obeys normal distribution does not exist. In case of this, the measured data will obey the normal distribution be adopted and the other measurement should be restricted. The Huber estimation divided the measure data to two classes. One is the normal data, which is used as traditional, and the other is the bad data which is used restrictedly, i.e., the influence of the bad data is reduced. Mathematically, the Huber estimation can be stated as follows.

$$
\rho\left(v_{i}\right)= \begin{cases}\frac{v_{i}^{2}}{2} & \left|v_{i}\right| \leq k \\ k\left|v_{i}\right|-\frac{k^{2}}{2} & \left|v_{i}\right|>k\end{cases}
$$

where $v_{i}$ is residual error, i.e., $v_{i}=\left(y_{c i}-y_{m i}\right) / s . \mathrm{y}_{c i}$ and $\mathrm{y}_{\mathrm{mi}}$ represent the calculated value and measured value, respectively. $\mathrm{s}$ is standard deviation when $\left|v_{i}\right| \leq k$, and $\mathrm{s}$ will take the value of median $\mathrm{K}_{\mathrm{MAD}}$ when $\left|v_{i}\right|>k, K_{M A D}=\operatorname{med}_{i}\left|y_{c i}-y_{m i}\right|$.

B. Robust Identification based on Huber Estimation

Based on the Huber estimation, the objective function of robust on-line identification for transmission line is,

$$
J(\alpha)=\sum_{i=1}^{N} P_{i}\left[\left.\frac{v_{i}^{2}}{2}\right|_{\left|v_{i}\right|<k \sigma_{0}}+\left.\left(k \sigma_{0}\left|v_{i}\right|-\frac{\left(k \sigma_{0}\right)^{2}}{2}\right)\right|_{k \sigma_{0} \leq v_{i} \mid}\right]
$$

where $\sigma_{0}$ is the standard error for voltage amplitude. For PMU $\sigma_{0}=0.002$.

\section{Simulation Results}

This section presents the comparisons of the traditional LS (TLS) and proposed robust LS method with Huber estimation (RLS) with the simulated data.

One $500 \mathrm{kV}$ transmission line is build in the PSCAD. The parameter is as follows. Length $=50 \mathrm{~km}$, $R=1.208 \Omega, X=19.803 \Omega, C=0.979$. In the simulation, $6000 \mathrm{PMU}$ data (time span=0.01s) is used.

In the case of with $0.5 \%$ Gauss noise in the line current and active/reactive power, the result of TLS and RLS are as shown in Table 1. Table 1 shows that in the case of with Gauss noise, the performance of the two approaches is almost same.

\begin{tabular}{|c|c|c|c|c|c|}
\hline Parameter & & & $R / \Omega$ & $X / \Omega$ & $\mathrm{C} / \mathrm{uF}$ \\
\hline Design Va & & & 1.208 & 19.80 & 0.979 \\
\hline & TI $\mathrm{T}$ & Result. & 1.207 & 19.79 & 0.9794 \\
\hline With 5\% & $1 \mathrm{LS}$ & Error\% & 0.083 & 0.05 & 0.04 \\
\hline Noise & DI $\mathrm{S}$ & Result. & 1.207 & 19.79 & 0.9794 \\
\hline & KLS & Error\% & 0.083 & 0.05 & 0.04 \\
\hline
\end{tabular}

Table 1 Results of LS and RLS Without/With Gaussian noise

Furthermore, in the case of 5\% of the PMU data is change to zero (become bad data) which may result from the data transfer error. The result of TLS and RLS with Huber are as shown in Table 2. Table 2 shows that, in the case of the data contain bad data, the error of RLS is smaller than the TLS. Thus, the RLS is more practical. 
Table 2 Results of LS and RLS under bad data

\begin{tabular}{lllll}
\hline \multicolumn{2}{l}{ Parameter } & $R / \Omega$ & $X / \Omega$ & $C / \mathrm{uF}$ \\
\hline \multirow{2}{*}{ TLS } & Result. & 2.338 & 30.23 & 1.103 \\
& Error\% & 93.70 & 52.75 & 12.66 \\
\multirow{2}{*}{ RLS } & Result. & 1.258 & 19.75 & 0.988 \\
& Error\% & 4.13 & 0.21 & 0.92 \\
\hline
\end{tabular}

\section{Conclusions}

This paper proposed a new robust to transmission line parameter estimation with Huber estimation. The proposed method formulates the identification of line parameter as an optimization problem with Huber estimation, which divided the measure data to two classes, one is the normal data, and the other is the bad data which is used restrictedly. The simulation results based on PSCAD demonstrate the effectiveness, noise immunity ability and robust ability of the proposed method.

\section{References}

[1] Ali Abur and Antonio Gomez Exposito, Power System State Estimation: Theory and Implementation, CRC Press, 2004

[2] LIU W, WU F, LUN S. Parameter error identification and estimation in power system state estimation. IEEE Trans on Power Systems, 1995, 10(1): 200-209.

[3] NING Liaoyi, SUN Hongbin, WU Wenchuan et al. State estimation based branch parameter estimation method for power grid. Proceedings of the CSEE, 2009, 29(1): 7-13.

[4] Wang M H, Bao J, et al. “Online estimation of transmission line parameters based on PMU measurements” Automation of Electric Power System, vol. 34, no. 1, pp. 25-27, Jan. 2010.

[5] Wilson R. E, Gary A, et al. Calculation of transmission line parameters from synchronized measurements. Electric Machines and Power Systems, 1999, 27(12): 1269-1278.

[6] Yuan L and Mladen K. "Optional Estimate of transmission line fault location considering measurement errors,” IEEE Trans. Power Delivery, vol 22, no. 3, pp. 1335-1341, July, 2007.

[7] Il-Dong Kim and Raj K. Aggarwal. "A study on the on-line measurement of transmission line impedances for improved relaying protection,” Electrical Power and Energy Systems, vol. 28, no. 6, pp. 359-366, 2006.

[8] Di Shi, Tylavsky, D.J., Logic, N., et al., "Identification of short transmission-line parameters from synchrophasor measurements," presented at the 40nd Int. Conf. North American Power Symposium, USA, 2008.

[9] Ding Lan. Study on parameter identification of transmission line and thevenin equivalent based on PMU. Beijing, north china electric power university, 2012.

[10]A. G. Phadke, "Synchronised phasor measurements - a historical overview”, in Proc. IEEE/PES Transmission and Distribution Conf. Exhib., Asia Pacific, pp 476-479,2000. 